\title{
Recent Borexino results and prospects for the near future
}

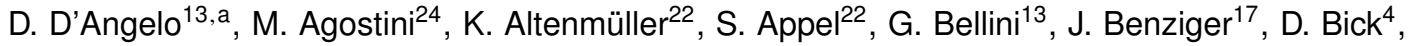
G. Bonfini ${ }^{12}$, D. Bravo ${ }^{20}$, B. Caccianiga $^{13}$, F. Calaprice ${ }^{16}$, A. Caminata ${ }^{3}$, P. Cavalcante ${ }^{12}$, A. Chepurnov $^{23}$, S. Davini ${ }^{24}$, A. Derbin ${ }^{15}$, L. Di Noto ${ }^{3}$, I. Drachnev ${ }^{24}$, A. Etenko ${ }^{10}$, K. Fomenko ${ }^{2}$, D. Franco ${ }^{1}$, F. Gabriele ${ }^{12}$, C. Galbiati ${ }^{16}$, C. Ghiano ${ }^{3}$, M. Giammarchi ${ }^{13}$, M. Goeger-Neff ${ }^{22}$, A. Goretti ${ }^{16}$, M. Gromov ${ }^{23}$, C. Hagner ${ }^{4}$, E. Hungerford ${ }^{25}$, Aldo lanni ${ }^{12}$, Andrea lanni ${ }^{16}$, K. Jedrzejczak ${ }^{6}$, M. Kaiser ${ }^{4}$, V. Kobychev ${ }^{7}$, D. Korablev², G. Korga ${ }^{12}$, D. Kryn ${ }^{1}$, M. Laubenstein ${ }^{12}$, B. Lehnert ${ }^{26}$, E. Litvinovich ${ }^{10,11}$, F. Lombardi ${ }^{12}$, P. Lombardi ${ }^{13}$, L. Ludhova ${ }^{13, b}$, G. Lukyanchenko ${ }^{10}$, I. Machulin ${ }^{10,11}$, S. Manecki ${ }^{20}$, W. Maneschg ${ }^{5}$, S. Marcocci ${ }^{24}, \mathrm{E}_{\text {. Meroni }}{ }^{13}$, M. Meyer ${ }^{4}$, L. Miramonti ${ }^{13}$, M. Misiaszek ${ }^{6,12}$, M. Montuschi ${ }^{21}$, P. Mosteiro ${ }^{16}$, V. Muratova ${ }^{15}$, B. Neumair ${ }^{22}$, L. Oberauer ${ }^{22}$, M. Obolensky ${ }^{1}$, F. Ortica $^{14}$, M. Pallavicini ${ }^{3}$, L. Papp ${ }^{22}$, L. Perasso $^{3}$, A. Pocar $^{19}$, G. Ranucci ${ }^{13}$, A. Razeto ${ }^{12}$, A. Re ${ }^{13}, A \cdot R o m a n i^{14}, R \cdot R o n c i n{ }^{12,1}, N$. Rossi ${ }^{12}$, S. Schönert ${ }^{22}$, D. Semenov ${ }^{15}$, H. Simgen ${ }^{5}$, M. Skorokhvatov ${ }^{10,11}$, O. Smirnov ${ }^{2}$, A. Sotnikov ${ }^{2}$, S. Sukhotin ${ }^{10}$, Y. Suvorov ${ }^{27,10}$, R. Tartaglia ${ }^{12}$, G. Testera ${ }^{3}$, J. Thurn ${ }^{26}$, M. Toropova ${ }^{10}$, E. Unzhakov ${ }^{15}$, A. Vishneva ${ }^{2}$, R.B. $V_{\text {ogelaar }}{ }^{20}$, F. von Feilitzsch ${ }^{22}$, H. Wang ${ }^{27}$, S. Weinz ${ }^{28}$, J. Winter ${ }^{28}$, M. Wojcik ${ }^{6}$, M. Wurm ${ }^{28}$, Z. Yokley ${ }^{20}$, O. Zaimidoroga ${ }^{2}$, S. Zavatarelli ${ }^{3}$, K. Zuber $^{26}$, and G. Zuzel ${ }^{6}$

${ }^{1}$ AstroParticule et Cosmologie, Université Paris Diderot, CNRS/IN2P3, CEA/IRFU, Observatoire de Paris, Sorbonne Paris Cité, 75205 Paris Cedex 13, France

${ }^{2}$ Joint Institute for Nuclear Research, 141980 Dubna, Russia

${ }^{3}$ Dipartimento di Fisica, Università degli Studi e INFN, Genova 16146, Italy

${ }^{4}$ Institut für Experimentalphysik, Universität, 22761 Hamburg, Germany

${ }^{5}$ Max-Planck-Institut für Kernphysik, 69117 Heidelberg, Germany

${ }^{6}$ M. Smoluchowski Institute of Physics, Jagiellonian University, 30059 Krakow, Poland

${ }^{7}$ Kiev Institute for Nuclear Research, $06380 \mathrm{Kiev}$, Ukraine

${ }^{8}$ Institute for Theoretical and Experimental Physics, 117218 Moscow, Russia

${ }^{9}$ Kepler Center for Astro and Particle Physics, Universität Tübingen, 72076 Tübingen, Germany.

${ }^{10}$ NRC Kurchatov Institute, 123182 Moscow, Russia

${ }^{11}$ National Research Nuclear University MEPhI (Moscow Engineering Physics Institute), 115409 Moscow,

Russia.

${ }^{12}$ INFN Laboratori Nazionali del Gran Sasso, 67010 Assergi (AQ), Italy

${ }^{13}$ Dipartimento di Fisica, Università degli Studi e INFN, 20133 Milano, Italy

${ }^{14}$ Dipartimento di Chimica, Università e INFN, 06123 Perugia, Italy

${ }^{15}$ St. Petersburg Nuclear Physics Institute NRC Kurchatov Institute, 188350 Gatchina, Russia

${ }^{16}$ Physics Department, Princeton University, Princeton, NJ 08544, USA

${ }^{17}$ Chemical Engineering Department, Princeton University, Princeton, NJ 08544, USA

${ }^{18}$ Physics Department, Queen's University, Kingston ON K7L 3N6, Canada

${ }^{19}$ Amherst Center for Fundamental Interactions and Physics Department, University of Massachusetts, Amherst, MA 01003, USA

\footnotetext{
${ }^{\mathrm{a}} \mathrm{e}$-mail: davide.dangelo@mi.infn.it

${ }^{b}$ Now at RWTH Aachen University, 52074, Aachen, Germany and Forschungzentrum Jülich IKP-2, 52428 Jülich, Germany
} 
${ }^{20}$ Physics Department, Virginia Polytechnic Institute and State University, Blacksburg, VA 24061, USA

${ }^{21}$ Dipartimento di Fisica e Scienze della Terra Università degli Studi di Ferrara e INFN, Via Saragat 1-44122,

Ferrara, Italy

${ }^{22}$ Physik-Department and Excellence Cluster Universe, Technische Universität München, 85748 Garching, Germany

${ }^{23}$ Lomonosov Moscow State University Skobeltsyn Institute of Nuclear Physics, 119234 Moscow, Russia

${ }^{24}$ Gran Sasso Science Institute (INFN), 67100 L'Aquila, Italy

${ }^{25}$ Department of Physics, University of Houston, Houston, TX 77204, USA

${ }^{26}$ Department of Physics, Technische Universität Dresden, 01062 Dresden, Germany

${ }^{27}$ Physics and Astronomy Department, University of California Los Angeles (UCLA), Los Angeles, California 90095, USA

${ }^{28}$ Institute of Physics and Excellence Cluster PRISMA, Johannes Gutenberg-Universität Mainz, 55099 Mainz, Germany

\begin{abstract}
The Borexino experiment located in the Gran Sasso National Laboratory, is an organic liquid scintillator detector conceived for the real time spectroscopy of low energy solar neutrinos. The phase-I of the data taking campaign $(2007-2010)$ has allowed the first independent measurements of ${ }^{7} \mathrm{Be}$ and pep solar neutrino fluxes as well as the first measurement of anti-neutrinos from the Earth. After a purification of the scintillator, Borexino is now in phase-II since 2011. Thanks to the unprecedented background levels, we have performed the first flux measurement of neutrinos from the fundamental pp reaction which powers the Sun. We review this breakthrough result and other recent results, including the latest review of our terrestrial neutrino analysis. We also discuss the upcoming measurements on middle energy solar neutrino spectral components (pep, $\mathrm{CNO}$ ) and the new project SOX devoted to the study of sterile neutrinos via the use of a neutrino source placed in close proximity of the detector's active material.
\end{abstract}

\title{
1 Why solar neutrinos with Borexino
}

The Sun is an intense source of neutrinos, produced in nuclear reactions of the p-p chain and of the CNO cycle [1]. Measurements of the individual neutrino fluxes is of paramount importance for

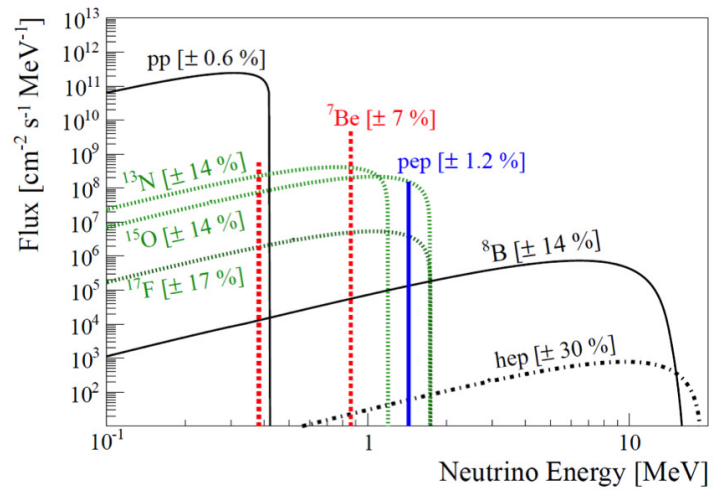

Figure 1. Solar Neutrino Spectrum.

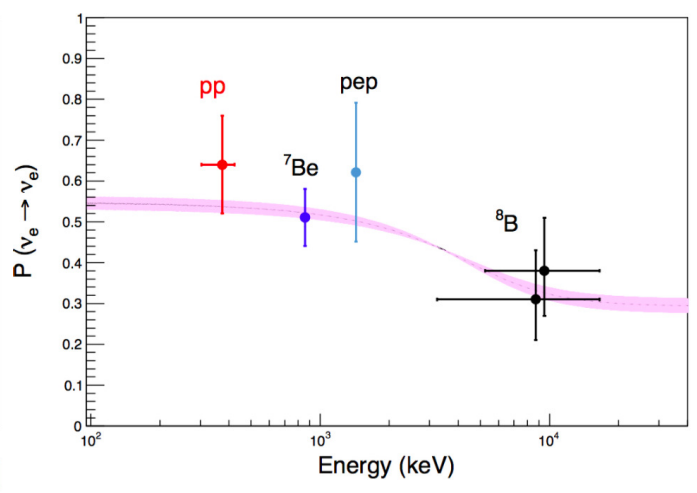

Figure 2. Survival probability of electron neutrinos as foreseen by the MSW-LMA model. Data points discussed here are also shown. 
both particle physics and astrophysics. The solar neutrino spectrum can be seen in figure 1. Up to a few years ago, spectroscopical measurements were performed by water Cherenkov detectors above $\sim 5 \mathrm{Mev}$ and concerned only ${ }^{8} \mathrm{~B}$ neutrinos for less then $1 \%$ of the total flux. The bulk of neutrinos at low energies were detected only with radiochemical experiments, incapable of resolving the individual components. Neutrinos are emitted in the Sun as electron-flavour neutrinos and may oscillate to a different flavour during the trajectory to the Earth. The MSW mechanism with Large Mixing Angle (LMA) foresees the survival probability for electron neutrinos on Earth after oscillation is taken into account, as shown in figure 2. Borexino was designed to perform spectroscopy of the low energy part of the solar neutrino spectrum, in particular the flux of the ${ }^{7} \mathrm{Be}$ monochromatic line at $862 \mathrm{keV}$. Borexino has largely exceeded the expected performance and broadened the physics program past the original goal.

\section{The Borexino Project}

Detector layout. The Borexino detector [2] is sketched in figure 3. It is located at the Gran Sasso National Laboratories (LNGS) in central Italy, at a depth of $3800 \mathrm{~m}$ w.e.. The active mass consists of 278 tons of pseudocumene (PC), doped with $1.5 \mathrm{~g} / \mathrm{l}$ of PPO. The scintillator is contained in a thin $(125 \mu \mathrm{m})$ nylon Inner Vessel (IV), $8.5 \mathrm{~m}$ in diameter. The IV is surrounded by two concentric PC buffers doped with a light quencher. The scintillator and buffers are contained in a Stainless Steel Sphere (SSS) with a diameter of $13.7 \mathrm{~m}$. The SSS is enclosed in a Water Tank (WT), containing 2100 tons of ultra-pure water as an additional shield against backgrounds from the laboratory environment. The scintillation light released upon particle interaction

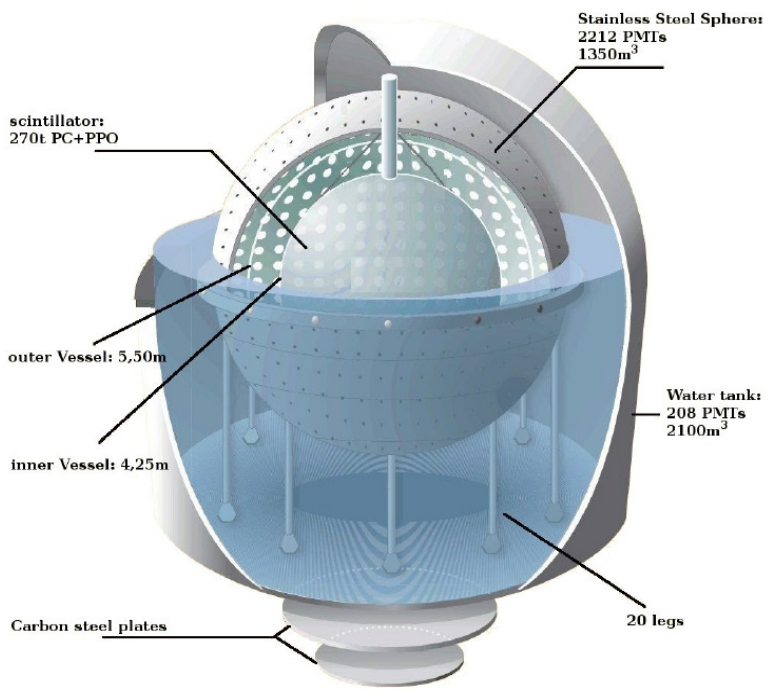

Figure 3. Sketch of the Borexino detector. in the pseudocumene is detected by 2212 8" PhotoMultiplier Tubes (PMTs) uniformly distributed on the inner surface of the SSS. Additional 208 8" PMTs instrument the WT and detect the Cherenkov light radiated by cosmic muons that cross the water shield.

Detector performance. The Borexino scintillator has a light yield of $\sim 10^{4}$ photons $/ \mathrm{MeV}$, resulting in $\sim 500$ detected photoelectrons/MeV. The fast time response $(\sim 3 \mathrm{~ns})$ of the scintillating mixture allows to reconstruct the events position by means of a time-of-flight technique with $\sim 13 \mathrm{~cm}$ precision for events at the center of the detector. Depending on the analysis, the fiducial volume is defined between 75 tons and 150 tons. Neutrinos interact via elastic scattering on the electrons of the target material. The signature of ${ }^{7} \mathrm{Be}$ neutrinos is a Compton-like shoulder at $665 \mathrm{keV}$ in the electron recoil spectrum. The energy resolution $(1 \sigma)$ at ${ }^{7} \mathrm{Be}$ energy is as low as $44 \mathrm{keV}$ (or $6.6 \%$ ). The inner detector trigger is defined as the coincident firing of at least 20-25 PMTs, corresponding to an energy threshold of $\sim 40-50 \mathrm{keV}$. However the analysis threshold in phase-I was limited by the knowledge 
Table 1. Background levels in the Borexino scintillator. cpd stands for "counts per day".

\begin{tabular}{ccccc}
\hline Isotope & Typical & Required & Before purification & After purification \\
\hline${ }^{238} \mathrm{U}$ & $210^{-5} \mathrm{~g} / \mathrm{g}$ (dust) & $\leq 10^{-16} \mathrm{~g} / \mathrm{g}$ & $(5.3 \pm 0.5) 10^{-18} \mathrm{~g} / \mathrm{g}$ & $<0.810^{-19} \mathrm{~g} / \mathrm{g}$ \\
${ }^{232} \mathrm{Th}$ & $210^{-5} \mathrm{~g} / \mathrm{g}$ (dust) & $\leq 10^{-16} \mathrm{~g} / \mathrm{g}$ & $(3.8 \pm 0.8) 10^{-18} \mathrm{~g} / \mathrm{g}$ & $<1.010^{-18} \mathrm{~g} / \mathrm{g}$ \\
${ }^{14} \mathrm{C} /{ }^{12} \mathrm{C}$ & $10^{-12}$ (cosmogenic) & $\leq 10^{-18}$ & $(2.69 \pm 0.06) 10^{-18}$ & unchanged \\
${ }^{222} \mathrm{Rn}$ & 100 atoms $/ \mathrm{cm}^{3}$ (air) & $\leq 10 \mathrm{cpd} / 100 \mathrm{t}$ & $1 \mathrm{cpd} / 100 \mathrm{t}$ & unchanged \\
${ }^{40} \mathrm{~K}$ & $210^{-6} \mathrm{~g} / \mathrm{g}$ (dust) & $\leq 10^{-18} \mathrm{~g} / \mathrm{g}$ & $\leq 0.410^{-18} \mathrm{~g} / \mathrm{g}$ & unchanged \\
${ }^{85} \mathrm{Kr}$ & $1 \mathrm{~Bq} / \mathrm{m}^{3}$ (air) & $\leq 1 \mathrm{cpd} / 100 \mathrm{t}$ & $(30 \pm 5) 10 \mathrm{cpd} / 100 \mathrm{t}$ & $\leq 5 \mathrm{cpd} / 100 \mathrm{t}$ \\
${ }^{39} \mathrm{Ar}$ & $17 \mathrm{mBq} / \mathrm{m}^{3}$ (air) & $\leq 1 \mathrm{cpd} / 100 \mathrm{t}$ & $\ll{ }^{85} \mathrm{Kr}$ & $\ll{ }^{85} \mathrm{Kr}$ \\
${ }^{210} \mathrm{Po}$ & & not specified & $\sim 80 \rightarrow \sim 5 \mathrm{cpd} / \mathrm{t}$ & unchanged \\
${ }^{210} \mathrm{Bi}$ & & not specified & $\sim 10 \rightarrow \sim 75 \mathrm{cpd} / 100 \mathrm{t}$ & $(27 \pm 8) \mathrm{cpd} / 100 \mathrm{t}$ \\
\hline
\end{tabular}

of the ${ }^{14} \mathrm{C}$ spectral shape to $\sim 250 \mathrm{keV}$. In phase-II we have successfully reduced our threshold to $165 \mathrm{keV}$ (see Sect. 4). Pulse Shape Analysis (PSA) is performed to identify various classes of events, among which electronic noise, pile-up events, muons, $\alpha$ and $\beta$ particles.

Borexino timeline. The phase-I of Borexino occurred between May 2007 and May 2010. Next, we proceeded to the purification of the scintillator by water extraction and sparging with a specially developed nitrogen gas, low in $\mathrm{Kr}$ and Ar contamination. After looping through the purification plant for six complete cycles, in November 2011 the phase-II of Borexino started and is expected to last at least until the end of 2016. The energy scale uncertainty in the range [0.2-2.0]MeV has been determined at $1.5 \%$ precision, using multiple gamma sources. The position reconstruction algorithm has been tuned using a $\mathrm{Rn}$ source placed in 184 positions inside the active volume. The determination of the fiducial volume is now $-1.3 \%+0.5 \%$. An external calibration campaign has allowed to correctly model external gamma backgrounds. The calibrations will be repeated before the end of phase-II to demonstrate stability of the detector and possibly to improve the previous results[3].

Experimental backgrounds. The background levels of the Borexino scintillator are summarised in table $1 .{ }^{238} \mathrm{U}$ and ${ }^{232} \mathrm{Th}$ contaminations are unprecedented and largely exceed the requirements of the experiment. Most other contaminants are at acceptable levels or have been reduced below required limits after purification. We observe the presence of Radon Daughters ${ }^{210} \mathrm{Bi}$ and ${ }^{210} \mathrm{Po}$ out of equilibrium. ${ }^{210} \mathrm{Po}$ is of little concern as it is an $\alpha$ emitter and it can be identified by PSA. ${ }^{210} \mathrm{Bi}$ contamination has been rising during the data taking of phase-I due to unclear motivations, possibly related to the movement of the scintillator. This variation was modelled and taken into account during the ${ }^{7} \mathrm{Be}$ flux modulation analysis (Sect. ??). ${ }^{210} \mathrm{~B}$ was later reduced by the purification campaign to a level which allowed the measurement of pp neutrino (Sect. 4) and will possibly allow the measurement of CNO neutrino fluxes (Sect. 7).

\section{Phase-I results}

${ }^{7}$ Be flux. The measurement of the flux of ${ }^{7} \mathrm{Be}$ neutrinos was the primary goal of Borexino. Thanks to the unprecedented background levels, the first observation was published in summer 2007, after only 3 months of data taking. The flux was later reviewed in 2008 and 2011 reducing the error every time. The signal is extracted by a spectral fit along with other neutrino signals and background components (figure 4). 
The fit is performed both using analytical spectral shapes and MonteCarlo generated curves, with or without the statistical subtraction of $\alpha$ events via PSA, obtaining the same result within errors. The last measured rate[4] after $741 \mathrm{~d}$ of live time (full phase-I) is $R_{\mathrm{Be}}=46.0 \pm 1.5_{\text {stat }} \pm 1.6_{\text {syst }} / \mathrm{d} / 100 \mathrm{t}$. For the first time the experimental error $(4.8 \%)$ is lower the theoretical error $(7 \%)$. The rate corresponds to a flux of $\Phi_{B e}=(3.10 \pm 0.15) \times$ $10^{9} \mathrm{~cm}^{-2} \mathrm{~s}^{-1}$ with a survival probability $P_{e e}=$ $0.51 \pm 0.07$ at $862 \mathrm{keV}$.

We have also carefully inspected the data set at the energy of ${ }^{7} \mathrm{Be}$ neutrino scattering looking for an eventual day-night asymmetry[5]. This was foreseen in an alternative MSW scenario, called LOW, also compatible to some extent with the solar neutrino results. The LOW scenario before Borexino could be excluded only assuming CPT invariance and using the KamLAND reactor's antineutrino results. Our data is consistent with no asymmetry: $A_{D N}=\Phi_{N}-\Phi_{D} / \Phi=0.001 \pm 0.012_{\text {stat }} \pm 0.007_{\text {syst }}$. With this result, the MSW-LOW mechanism can be ruled out at $8.5 \sigma$ using only solar neutrino data.

${ }^{8} \mathbf{B}$ flux Borexino has measured the ${ }^{8} \mathrm{~B}$ flux down to $3.0 \mathrm{MeV}[6]$. While the much larger water Cherenkov detectors can achieve better precision, Borexino holds the lowest threshold achieved so far. Lowering the threshold on ${ }^{8} \mathrm{~B}$ spectroscopy represents one of the key features to inspect the transition region of the LMA scenario (see Sect. 1 and 4). The measured rate is $R_{B}=0.22 \pm 0.04_{\text {stat }} \pm 0.01_{\text {syst }}$ and corresponds to a flux of $\Phi_{B}=\left(2.4 \pm 0.4_{\text {stat }} \pm 0.1_{\text {syst }}\right) \times 10^{6} \mathrm{~cm}^{-2} \mathrm{~s}^{-1}$. The flux above $5 \mathrm{MeV}$ is in good consistency with other results.

pep flux and CNO limits. As shown in figure 2, the pep neutrino energy lies at the boundary between the Vacuum and the Transition region of the MSW survival probability. Neutrinos from the pep reaction are closely related to the fundamental pp neutrinos and have their flux theoretically well constrained by this relation. In the same energy region are neutrinos from the $\mathrm{CNO}$ cycle reactions. CNO neutrinos are poorly constrained by the SSM and have never been detected so far. At this energy the cosmogenic background of ${ }^{11} \mathrm{C}$ is overwhelming the pep/CNO neutrino flux by about an order of magnitude. We have made the measurement[7] possible suppressing this background exploiting the three-fold coincidence between the parent muon, the ${ }^{11} \mathrm{C}$ and the neutron most often accompanying its production. By excluding the regions of the active volume close to the muon track or to the neutron capture point for a few half lives of ${ }^{11} \mathrm{C}(29 \mathrm{~min})$, we select about $50 \%$ of the exposure where the ${ }^{11} \mathrm{C}$ background is only $10 \%$ of the total. Another powerful handle to distinguish neutrinos from is the pulse shape. The annihilation energy accompanying the ${ }^{11} \mathrm{C} \mathrm{e}^{+}$-decay may be delayed when released by ortho-positronium bound state. The rate of pep neutrinos has been extracted with a multivariate analysis based of the energy of the event, the distance from the center of the detector and a pulse shape parameter[8]. The rate is $R_{\text {pep }}=\left(3.1 \pm 0.6_{\text {stat }} \pm 0.3_{\text {syst }}\right) / \mathrm{d} / 100 \mathrm{t}$ corresponding to a flux of $\Phi_{\text {pep }}=(1.6 \pm 0.3) \times 10^{8} \mathrm{~cm}^{-2} \mathrm{~s}^{-1}$ and a survival probability of $P_{\text {pep }}^{e e}=0.62 \pm 0.17$ at $1.44 \mathrm{MeV}$. The flux of $\mathrm{CNO}$ neutrinos could not be extracted due to the spectral shape degeneracy with the ${ }^{210} \mathrm{Bi}$ background. The strongest upper limit available to date has been however obtained 

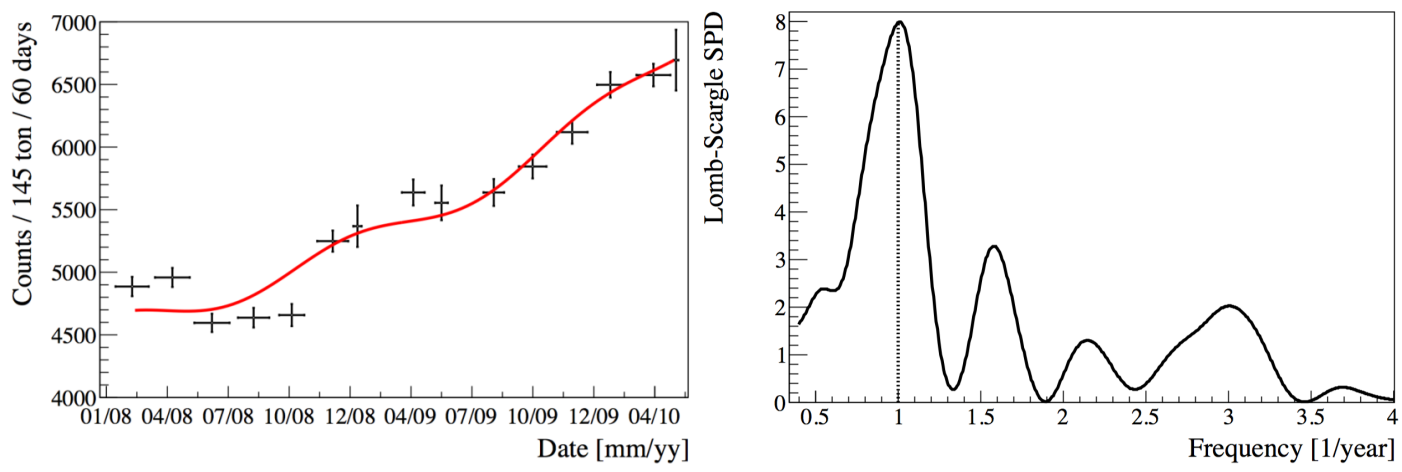

Figure 5. ${ }^{7}$ Be flux modulation analysis. Left: ' $60 \mathrm{~d}$ '-binned count rate evolution. Right: ' $10 \mathrm{~d}$ '-binned LombScargle Spectral Power Density.

from this analysis. The rate of CNO neutrinos is $R<7.1 / \mathrm{d} / 100 \mathrm{t}$ at $95 \%$ C.L. corresponding to $\Phi_{\mathrm{CNO}}<7.7 \times 10^{8} \mathrm{~cm}^{-2} \mathrm{~s}^{-1}$. Improving this analysis using the phase-II data to reduce errors and possibly detect CNO neutrinos is our main goal for 2016 .

Annual modulation. Due to the eccentricity of the Earth's orbit, the observed solar neutrino flux is expected to undergo a yearly modulation with amplitude $\pm 3.4 \%$ and a maximum at the beginning of January. The presence of such modulation in the ${ }^{7} \mathrm{Be}$ flux is the ultimate proof that Borexino is actually observing neutrinos from the Sun. For this analysis[8] we have defined a dynamic and enlarged $141 \mathrm{t} \mathrm{FV}$. This has been possible thanks to the precise determination of the vessel's shape from the distribution of ${ }^{210} \mathrm{Bi}$ events deposited on the vessel's surface on a weekly basis. Nevertheless performing the spectral fit on sub-periods of the data set is not a viable method due to reduced statistics. Using the phase-I data set ( $850 \mathrm{~d}$ astr. time), we have considered the count rate in the ${ }^{7}$ Be region (105-380 p.e.), which also includes background from the decay of ${ }^{210} \mathrm{Bi}$ in the scintillator. The last has been rising exponentially during the phase-I (Sect. 2). We have averaged the rate on a $60 \mathrm{~d}$ base (figure 5, left) and we have fitted the result with:

$$
R=R_{0}+R_{B i} e^{\Lambda_{B i} t}+\bar{R}\left[1+2 \epsilon \cos \left(\frac{2 \pi t}{T}-\phi\right)\right]
$$

where $R_{0}$ accounts for a time independent background component. The rate $R_{B i}$ and the time constant $\Lambda_{B i}$ of ${ }^{210} \mathrm{Bi}$ background are fixed to values independently determined from a different energy interval. The period found is $T=(1.01 \pm 0.07) \mathrm{y}$ and the phase, measured from Jan $1^{\text {st }} 2008$, is $\phi=(11.0 \pm 4.0) \mathrm{d}$. The average ${ }^{7} \mathrm{Be}$ rate and the eccentricity $\epsilon$ are consistent within $2 \sigma$ with the spectral fit result and with the expected orbit eccentricity, respectively. The hypothesis of no modulation is rejected at $>3 \sigma$. An alternative approach uses Lomb-Scargle frequency analysis in $10 \mathrm{~d}$ binned data set. The Spectral Power Density (SPD) distribution is shown in figure 5 (right). The significance of SPD peaks has been evaluated by Monte Carlo simulations of the signal and the background. The peak at $1 \mathrm{y}$ with $\mathrm{SPD}=7.96$ has significance largely above $3 \sigma$, while no other peak exceeds $2 \sigma$. 


\section{4 pp neutrino flux}

Neutrino from the fundamental pp reaction which powers the Sun are the most elusive to detection as their energy only spans up to $420 \mathrm{keV}$, corresponding to $264 \mathrm{keV}$ in the recoil electron energy spectrum. The expected rate is $(131 \pm 2) / \mathrm{d} / 100 \mathrm{t}$. Our efforts to reduce the analysis threshold and successfully fit this part of the spectrum have been twofold. First, we performed the purification campaign described in Sect. 2: ${ }^{85} \mathrm{~K}$ was reduced to a level consistent with zero, ${ }^{210} \mathrm{Bi}$ by a factor $\sim 3$. Second, a large analysis effort has been done to understanding the overwhelming intrinsic background due to ${ }^{14} \mathrm{C}$ that extend up to $\sim 220 \mathrm{keV}$. Though the Borexino scintillator was obtained from selected ancient oil batches to have a low ${ }^{14} \mathrm{C}$ content, the detector's trigger rate of $\sim 30 \mathrm{~s}^{-1}$ ( $\sim 50 \mathrm{keV}$ hardware threshold) is largely dominated by ${ }^{14} \mathrm{C}$ decays. Constraining the rate and correctly modelling the shape of ${ }^{14} \mathrm{C}$ was hard due to effect of the threshold. We overcame this problem studying the events that accidentally fall within the $16 \mu \mathrm{s}$ acquisition window opened by another uncorrelated event. The ${ }^{14} \mathrm{C}$ rate was thus measured to be $(40 \pm 1) / \mathrm{s} / 100 \mathrm{t} .{ }^{14} \mathrm{C}$ gives a second problem: pile-up. Due to the high rate, we record events arising from a ${ }^{14} \mathrm{C}$ decay overlapped with ${ }^{14} \mathrm{C},{ }^{210} \mathrm{Po}$, dark noise from the PMTs and virtually any other spectral species. The spectral shape for this class of events is unknown and the position reconstruction largely fails. We have therefore generated a synthetic pile-up sample, overlapping uncorrelated events with regular data. The obtained energy spectrum is used in the spectral fit and the rate is constrained within errors to the value obtained by this independent determination. Using data from January 2012 to May 2013 ( $408 \mathrm{~d}$ live time), we obtained the first direct detection of pp neutrinos. The fiducial volume was set to $75 \mathrm{t}$ for a resulting exposure of $\sim 85 \mathrm{ton} \cdot \mathrm{yr}$ We performed the fit in the $[165,590] \mathrm{keV}$ energy range as shown in figure 6 . The pp neutrino rate was determined to be:

$$
R_{p p}=144 \pm 13_{\text {stat }} \pm 10_{\text {sys }} / \mathrm{d} / 100 \mathrm{t}
$$

rejecting the null hypothesis at $10 \sigma$. The systematic error arises from the uncertainty in the energy estimator, in the fit energy range, in the data selection, in the pile-up evaluation and in the fiducial mass. The result interpretation is threefold: (i) assuming the SSM is correct, our result indicates a survival probability of $(0.64 \pm 0.12)$ in confirmation of neutrino oscillations with MSW-LMA mechanism in the vacuum dominated region; (ii) assuming the neutrino oscillation model is correct, our result converts to an unoscillated solar neutrino flux of $(6.6 \pm 0.7) \times 10^{10} \mathrm{~cm}^{-2} \mathrm{~s}^{-1}$, confirming the SSM predictions on neutrinos emitted by the reaction most fundamentally bound to the energy production in the Sun; (iii) if both previous assumptions are made, the Borexino result says that the luminosity of the Sun in neutrinos matches the photon luminosity, which is a proof of the Sun stability over a period of $10^{5}$ years. The measurement of the pp neutrino flux by Borexino was published by the Nature Journal[9]. 

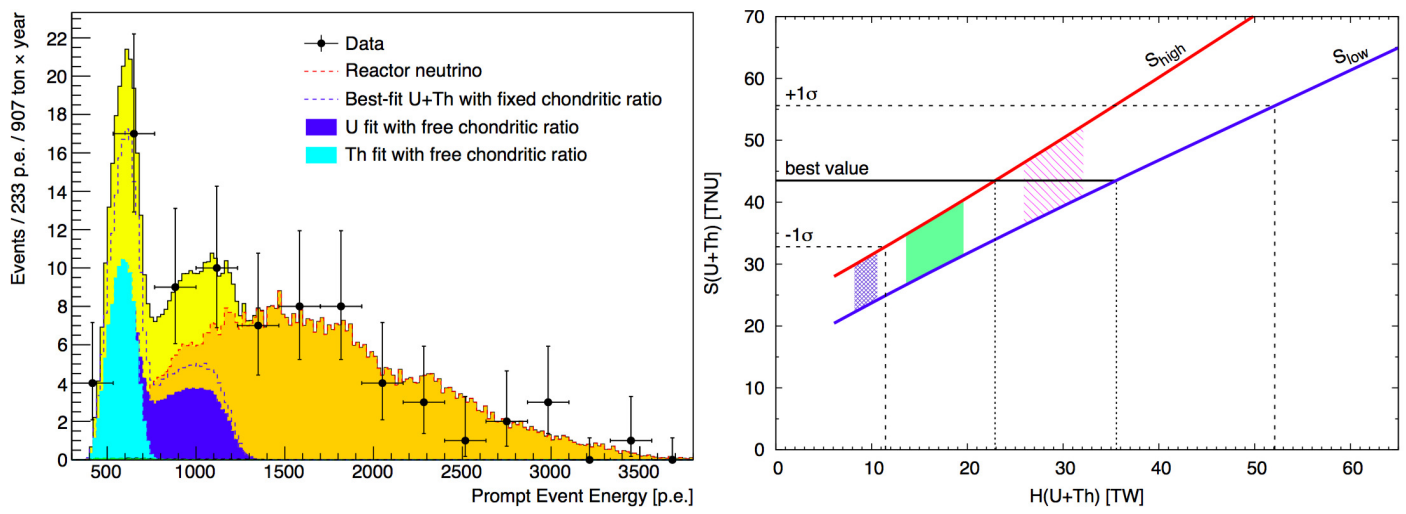

Figure 7. Left: antineutrino spectrum (prompt positron scattering). Right: Geoneutrino flux and terrestrial Heat generation foreseen by three different BSE models (coloured shaded areas). $S_{\text {high }}$ and $S_{\text {low }}$ are the limits where radiogenic material is concentrated at the Mantle-Crust and Core-Mantle boundary, respectively.

\section{Survival probability after Borexino.}

In figure 2, already introduced in Sect. 1, one can also see all the results of the Borexino low energy neutrino spectroscopy that we have discussed here, superimposed to the survival probability of solar electron neutrinos. The confirmation of the MSW-LMA model over a wide range of energies is striking. However, the unexplored transition region $(1-3 \mathrm{MeV})$ still has room for alternative models and new physics, in particular the proposed Non Standard neutrino Interactions (NSI) which foresee a different transition shape. There are two ways to test these hypotheses or confirm MSW-LMA: (i) reducing the errors on pep flux (and to a lower extent on ${ }^{7} \mathrm{Be}$ flux) and (ii) lowering the threshold on ${ }^{8} \mathrm{~B}$ neutrinos to observe (or not) the expected upturn of the spectrum. The analyses we are currently performing on Borexino Phase-II data follows both approaches.

\section{Geo-neutrinos}

Geo-neutrinos are anti-neutrinos produced by elements in the radioactive chains of ${ }^{238} \mathrm{U},{ }^{232} \mathrm{Th}$ and by the decay of ${ }^{40} \mathrm{~K}$, with a flux of the order of $\sim 10^{6} \mathrm{~cm}^{-2} \mathrm{~s}^{-1}$. These long lived elements are found in the Earth crust and mantel with unknown abundances. Earth models attribute about 19 out $44 \mathrm{TW}$ of thermal heat released by the Earth to radioactive decays of these elements. Measuring Geo-neutrinos flux at different locations can help the development of Earth models and shed light on the heat prodction mechanism of the planet. Only geo-neutrinos from ${ }^{238} \mathrm{U}$ and ${ }^{232} \mathrm{~T}$ chain elements can be measured by neutrino detectors, via Inverse Beta Decay with a threshold of $1.8 \mathrm{MeV}$. The ratio of $\mathrm{Th} / \mathrm{U}$ is supposed to be $\sim 3.9$ from the analysis of chondrites, meteorites with the same composition of the Earth. After the first observation in 2010 and a revision in 2013, we present here the most recent results obtained with a statistics of $2056 \mathrm{~d}[10]$. The main background component are the antineutrinos from power reactors, which we have carefully modelled from the fuel composition and up-time data provided by the power companies. Almost all other backgrounds are negligible, thanks to the tagging of the events given by the coincidence between the prompt positron scattering and the $\sim 250 \mu \mathrm{s}$-delayed $2.2 \mathrm{Mev}$ gamma from the $\mathrm{n}$ capture on $\mathrm{H}$. Events pairs are selected by energy cuts on both events and by their space-time correlation. The efficiency of the cuts is $(0.84 \pm 0.01)$ from simulations. We apply an en- 

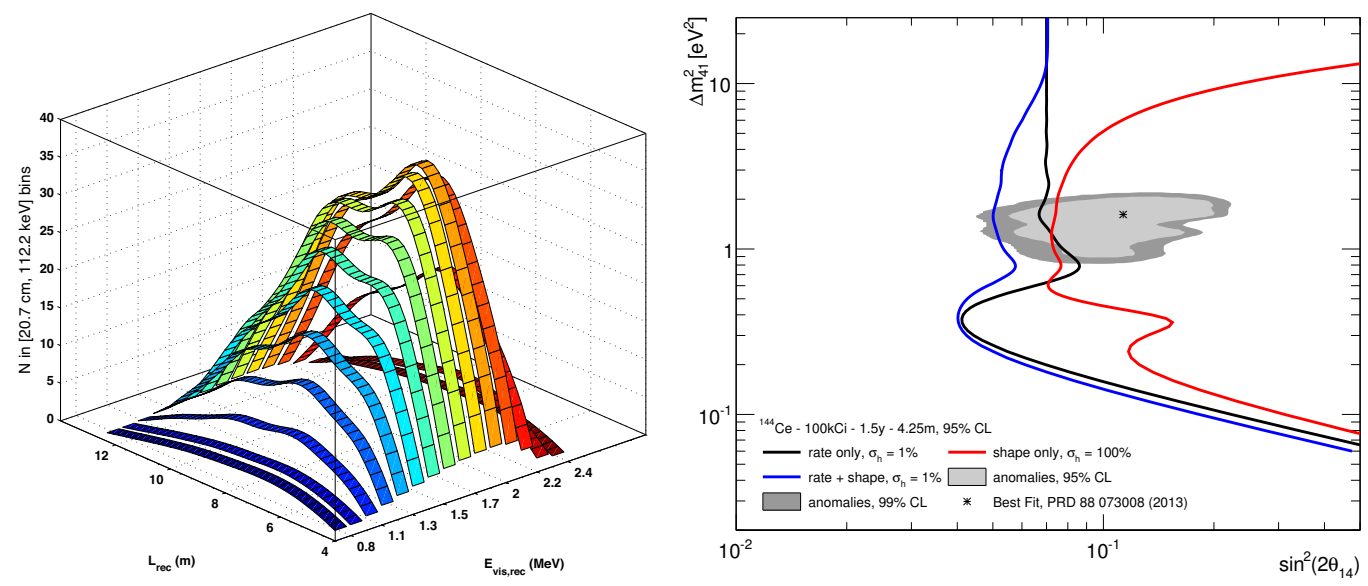

Figure 8. Oscillation wiggles in the SOX signals for a $100 \mathrm{kCi}{ }^{144} \mathrm{Ce}^{1144} \mathrm{Pr}$ source (left). Sox sensitivity (right).

larged dynamic fiducial volume that selects all the scintillator volume, excluding the outermost $30 \mathrm{~cm}$ to the IV surface with an average mass of $225 \mathrm{t}$ selected. The total exposure is $(907 \pm 44)$ ton $\cdot y r$ where we select 77 golden coincidences. The prompt event energy spectrum can be seen in figure 7 (left). An unbinned maximum likelihood fit returns:

$$
\begin{aligned}
N_{\text {geo }} & \left.=23.7_{-5.7}^{+6.5}(\text { stat })_{-0.6}^{+0.9} \text { (syst }\right) \\
N_{\text {react }} & \left.=52.7_{-7.7}^{+8.5}(\text { stat })_{-0.9}^{+0.7} \text { (syst }\right)
\end{aligned}
$$

The non observation of geo-neutrinos is excluded at $5.9 \sigma$ level. The corresponding fluxes are $S_{\text {geo }}=$ $(43.5 \pm 11.1) \mathrm{TNU}$ and $S_{\text {react }}=(96.6 \pm 15.9) \mathrm{TNU}$ where $1 \mathrm{TNU}=1 v / 10^{32}$ protons $/ \mathrm{yr}$. The flux of reactor antineutrinos compares well with the prediction of $(87 \pm 4) \mathrm{TNU}$. Freeing the $\mathrm{Th} / \mathrm{U}$ ration we can resolve the two components, obtaining overall similar results and a ratio in good agreement with the chondritic ratio. We account for the local contribution of the crust $N_{\mathrm{LOC}}=(9.7 \pm 1.3) \mathrm{TNU}$ obtained by a a geological survey of the area around LNGS and the contribution of the Rest Of the Crust $S_{\mathrm{ROC}}=(13.7 \pm 2.5) \mathrm{TNU}$ as predicted by the planetary model. We can then exclude the absence of a Mantle contribution at $98 \%$ C.L. and assign $S_{\text {Mantel }}=20.9_{-10.3}^{+15.9}$ TNU. Our results are in good agreement with the Bulk Silicate Earth model predictions as it can be seen in figure 7 (right).

\section{Short distance Oscillation with boreXino (SOX)}

In the past years many different experimental indications have pointed toward the existence of sterile neutrinos. Although none of them is individually strong enough to make a claim, altogether they justify a serious investigation. The hypothesised oscillation into the forth species occurs with $L / E \sim$ $1 \mathrm{~m} / \mathrm{MeV}$. In Borexino, using a neutrino source with energy of $\sim 1 \mathrm{MeV}$, the oscillation length is significantly smaller then the detector size $(\sim 10 \mathrm{~m})$ and significantly larger then the detector resolution $(\sim 12-15 \mathrm{~cm})$. This would allow to see oscillation wiggles in the position distribution of events, a powerful addition to a plain disappearance analysis. The location for such a source is the $\sim 1 \mathrm{~m}$ cubical pit present under the detector, which was excavated for this purpose before the detector's construction. 
This non invasive deployment requires no work on the detector, it bears no risk of contamination and does not terminate the solar run of Borexino. We foresee to deploy a ${ }^{144} \mathrm{Ce}^{144} \mathrm{Pr}$ source in November 2016 and proceed to a $1.5 \mathrm{y}$ data taking. ${ }^{144} \mathrm{Ce}-{ }^{144} \mathrm{Pr}$ is a $\beta$ emitter of antineutrinos with energies up to $3 \mathrm{MeV}$ and a decay time of $\sim 411 \mathrm{~d}$. Thanks to the neutron tagging, which makes antineutrino detection essentially background free (see Sect. 5), we can perform the measurement with a source activity of about $100-120 \mathrm{kCi}$, currently being produced at the Mayak facility (Russia). Figure 8 shows the expected signal in case of oscillation and the sensitivity of the experiment in the oscillation parameters' plane. Disappearance and wave effects will allow to clarify the matter, unambiguously proving or rejecting the hypothesis. In particular, in case of the existence of a fourth sterile neutrino with the parameters indicated, SOX[11] will surely discover the effect and measure the parameters of oscillation.

\section{Conclusions and outlook}

Borexino has performed low energy solar neutrino spectroscopy including ${ }^{7} \mathrm{Be}$, pep, ${ }^{8} \mathrm{~B}$ and pp neutrino fluxes. Thanks to its unprecedented background levels, which have been further reduced with a dedicated campaign of purification of the liquid scintillator, the phase-II data of Borexino currently ongoing will allow us to reduce the error on ${ }^{7} \mathrm{Be}$, pep and ${ }^{8} \mathrm{~B}$ neutrino flux measurements. Measurement of the so far undetected neutrinos from the CNO cycle reactions is of fundamental astrophysical importance in particular as the they can help resolve the solar metallicity puzzle[1]. The measurement requires an independent evaluation of the residual contamination of ${ }^{210} \mathrm{Bi}$ whose spectral shape is quasi degenerate with the $\mathrm{CNO}$ neutrinos. One way to do this is to infer the ${ }^{210} \mathrm{Bi}$ rate from its daughter ${ }^{210} \mathrm{Po}$, provided equilibrium can assumed. This assumption holds if there are no convective motions in the scintillator that may bring ${ }^{210}$ Po from the nylon vessel surface into the fiducial volume. We have recently installed a thermal insulation of the detector that should prevent such motion. If this will be successful we can proceed to measure the flux of CNO neutrinos, otherwise we will sensibly improve the limits we have already posed and we will consider a further purification campaign to reduce the ${ }^{210} \mathrm{Bi}$ contamination. At the end of phase-II we also expect to further improve our result on geo-neutrinos and to measure the seasonal variation effect of ${ }^{7} \mathrm{Be}$ neutrinos upon several cycles and without the background constraints of phase-I. Starting with November 2016 we will test the sterile neutrino hypothesis using a $100 \mathrm{kCi}{ }^{144} \mathrm{Ce}^{144} \mathrm{Pr}$ source placed under the detector.

\section{References}

[1] W.C. Haxton, R.G. Hamish Robertson and A. M. Serenelli, Ann. Rev. Astron. Astrophys. 51, 21 (2013).

[2] G. Bellini et al. (Borexino coll.), Nucl. Instrum. Methods A 609, 58 (2009).

[3] G. Bellini et al. (Borexino coll.), JINST 07, 10018 (2012).

[4] G. Bellini et al. (Borexino coll.), Phys. Rev. Lett. 107, 141302 (2011).

[5] G. Bellini et al. (Borexino coll.), Phys. Lett. B 707, 22 (2012).

[6] G. Bellini et al. (Borexino coll.), Phys. Rev. D 82, 033006 (2010).

[7] G. Bellini et al. (Borexino coll.), Phys. Rev. Lett. 108, 051302 (2012).

[8] G. Bellini et al. (Borexino coll.), Phys. Rev. D 89, 112007 (2014)

[9] G. Bellini et al. (Borexino coll.), Nature 512, 383-386 (2014).

[10] M. Agostini et al. (Borexino coll.), Phys. Rev. D 92, 031101(R) (2015).

[11] G. Bellini et al. (Borexino coll.), JHEP 08, 038 (2013). 